\title{
Patient with Huge Upper Esophageal Hemangioma
}

\author{
${ }^{1}$ Uri Alkan, ${ }^{2}$ Alex Geller, ${ }^{3}$ Jacob Shvero
}

\section{ABSTRACT}

Background: Cervical esophageal hemangioma is a rare benign tumor. Symptoms may include hematemesis, dyspnea, cough, dysphagia, and chest pain.

Objectives: To describe a case of a large esophageal hemangioma in order to raise awareness of the condition and highlight the use of a cooperative endoscopic treatment approach by a gastroenterologist and otolaryngologist with excellent results. The literature on large esophageal hemangioma is reviewed.

Materials and methods: An otherwise healthy male presented with a huge hemangioma of the upper esophagus. Imaging showed the mass hanging on a narrow stalk attached to the posterior wall of the cricopharyngeus muscle, filling the lumen of the cervical and mediastinal esophagus. Resection was accomplished with a combination of flexible gastroscopy and microscope-assisted rigid direct laryngoscopy.

Results: Pathologic evaluation showed a polypoid mass covered with squamous epithelium, with multiple engorged blood vessels. Some of the vessels were wide, surrounded by a thin wall, and others were narrow with a thick wall. The histology was compatible with arteriovenous hemangioma. There was no evidence of malignancy. At the 7-month postoperative follow-up, the patient appeared well, with no dyspnea or dysphagia. Our literature search yielded only two published cases of a huge hemangioma of the upper esophagus, which were treated by open resection. Ours is the first report of the use of endoscopic excision.

Conclusion: Large esophageal hemangioma should be part of the differential diagnosis of dyspnea, dysphagia, persistent cough, or chest pain. A combined approach by a gastroenterologist and otolaryngologist, working cooperatively, can facilitate the correct diagnosis and provide optimum treatment.

Keywords: Combined approach, Cough, Esophagus, Hemangioma.

How to cite this article: Alkan U, Geller A, Shvero J. Patient with Huge Upper Esophageal Hemangioma. Int J Otorhinolaryngol Clin 2017;9(1):25-27.

\section{Source of support: Nil}

\section{Conflict of interest: None}

\footnotetext{
${ }^{1}$ Resident, ${ }^{2}$ Assistant Professor, ${ }^{3}$ Professor

1,3 Department of Otorhinolaryngology Head and Neck Surgery Rabin Medical Center, and Sackler Faculty of Medicine, Tel Aviv University, Tel Aviv, Israel

${ }^{2}$ Department of Gastroenterology, Rabin Medical Center, and Sackler Faculty of Medicine, Tel Aviv University, Tel Aviv, Israel

Corresponding Author: Uri Alkan, Resident, Department of Otorhinolaryngology Head and Neck Surgery, Rabin Medical Center, and Sackler Faculty of Medicine, Tel Aviv University Tel Aviv, Israel, Phone: +91-97239376456, e-mail: urialkan@ gmail.com
}

\section{INTRODUCTION}

Hemangiomas are benign, endothelial tumors containing abnormal blood vessels. They account for $60 \%$ of all vascular tumors of the head and neck region. In adults, the supraglottis is the most common site. ${ }^{1}$ Esophageal and laryngeal hemangiomas are rare, ${ }^{2}$ although their reported prevalence has been rising with the increasing use of endoscopic examinations. ${ }^{3}$ Glottic and esophageal hemangiomas can cause massive bleeding, dysphagia, and dyspnea with airway compromise. ${ }^{4-6}$ Many cases, however, remain asymptomatic., Esophageal hemangiomas can also be incidental findings. ${ }^{4,6}$

Esophageal hemangioma is diagnosed by at least one of the following means: Computed tomography (CT), magnetic resonance imaging (MRI), barium esophagography, endoscopic examination, or ultrasonography of the neck. On CT scans, esophageal hemangiomas commonly appear as a well-outlined homogeneous lesion sometimes containing microcalcifications. ${ }^{7}$ T1-weighted MRI typically shows an isointense signal; on T2-weighted images, a high signal may indicate continuity with major blood vessels. ${ }^{7}$ Endoscopic examination reveals a prominent, red-blue, compressible submucosal mass. ${ }^{4,5,8}$

In the last few years, the treatment of esophageal hemangiomas has evolved from open surgery, such as esophagectomy, tumor enucleation, and sclerotherapy, to endoscopic intervention. ${ }^{3}$

The aim of this report was to describe an adult patient with a very unusual large arteriovenous esophageal hemangioma.

\section{CASE REPORT}

A 52-year-old otherwise healthy Caucasian male presented to the emergency department of a major tertiary medical center with complaints of cough and pressing chest pain of 1 month duration. There were no airway or gastrointestinal symptoms. The patient did not smoke, and he had no contributory family history. Detailed physical examination yielded no pathologic findings. Results of complete blood count, blood chemistry, electrocardiography, and chest $x$-ray were normal or negative. Chest $\mathrm{CT}$, performed as part of the comprehensive workup, revealed a cylindrical mass in the upper esophagus, $14 \mathrm{~cm}$ long by $2.5 \mathrm{~cm}$ wide (Fig. 1). It appeared to be hanging on a narrow stalk attached to the posterior wall of the cricopharyngeus muscle (upper esophageal 


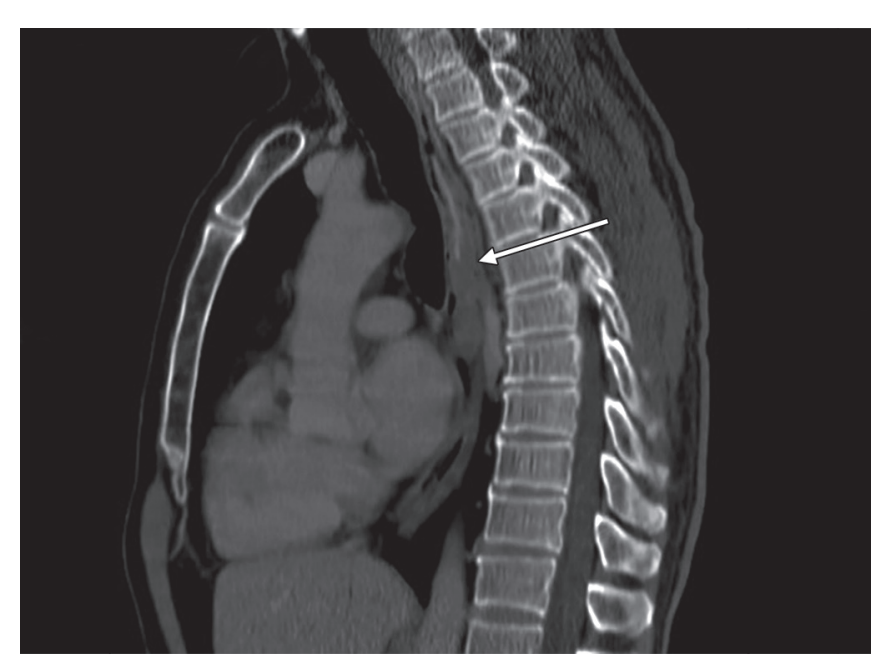

Fig. 1: Coronal reconstruction of the CT scan showing the mass filling the esophageal lumen

sphincter), extending from the hypopharynx cranially and filling the lumen of the upper and middle esophagus.

Gastroscopy revealed a mobile polypoid structure with normal overlying mucosa extending from the upper to the middle esophagus with an overall length of about $17 \mathrm{~cm}$. Biopsy study showed normal esophageal mucosa. On endoscopic ultrasound, the polypoid structure had a normal-appearing wall structure and a mixed echogenic pattern with multiple small vessels. No adenopathy was identified.

The patient was then referred to the otolaryngology department. On flexible endoscopy, a protrusion of the posterior aspect of the lower laryngeal wall was noted. The pyriform sinuses could not be visualized. There was no salivary retention or neck lymphadenopathy.

A multidisciplinary panel was convened to determine the optimal treatment approach. Removal of the mass was deemed necessary; open surgery was excluded because of its invasiveness and risks. A combined gastroscopic

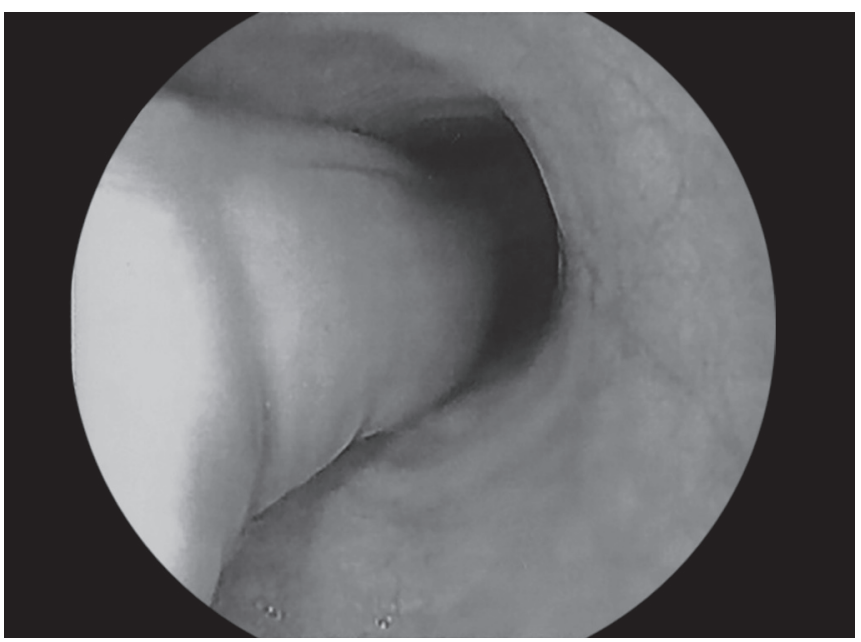

Fig. 2: View of the mass filling the esophagus at the gastroscopic examination and laryngoscopic approach was chosen. The procedure and risks were explained to the patient, who provided written informed consent.

The patient was placed under general anesthesia, and the invasive endoscopist inserted a flexible therapeutic video gastroscope (GIF 1T, Olympus, Japan) to visualize the mass (Fig. 2). Using endoscopic forceps, the endoscopist grasped the distal part of the mass and extracted it through the patient's mouth while the proximal part was still attached to the upper esophagus (Fig. 3). At this point, the otolaryngologist introduced a rigid esophagoscope. The stalk attaching the mass to the upper esophagus was identified, and the mass was separated with a harmonic scalpel.

Pathologic evaluation showed a polypoid mass covered with squamous epithelium, with multiple engorged blood vessels. Some of the vessels were wide and surrounded by a thin wall, and others were narrow with a thick wall. The histology was compatible with arteriovenous hemangioma. There was no evidence of malignancy.

At the 7-month postoperative follow-up, the patient appeared well, with no dyspnea or dysphagia.

\section{DISCUSSION}

In 1976, Feist et $\mathrm{al}^{7}$ were the first to describe a giant $(12 \times 5 \mathrm{~cm})$ hemangioma of the proximal esophagus. More than 30 years later, Kim et $\mathrm{al}^{5}$ reported a similar case of an $8.5 \times 6.5 \times 4 \mathrm{~cm}$ hemangioma of the distal esophagus. Both these tumors were submucosal and attached to the esophageal wall by a thin stalk, as in the present case. However, unlike the hemangioma described here, they were resected by major open surgery. Our search of the literature yielded no previous cases of endoscopic excision of a large esophageal hemangioma.

Notwithstanding its rarity, large esophageal hemangioma should be part of the differential diagnosis of

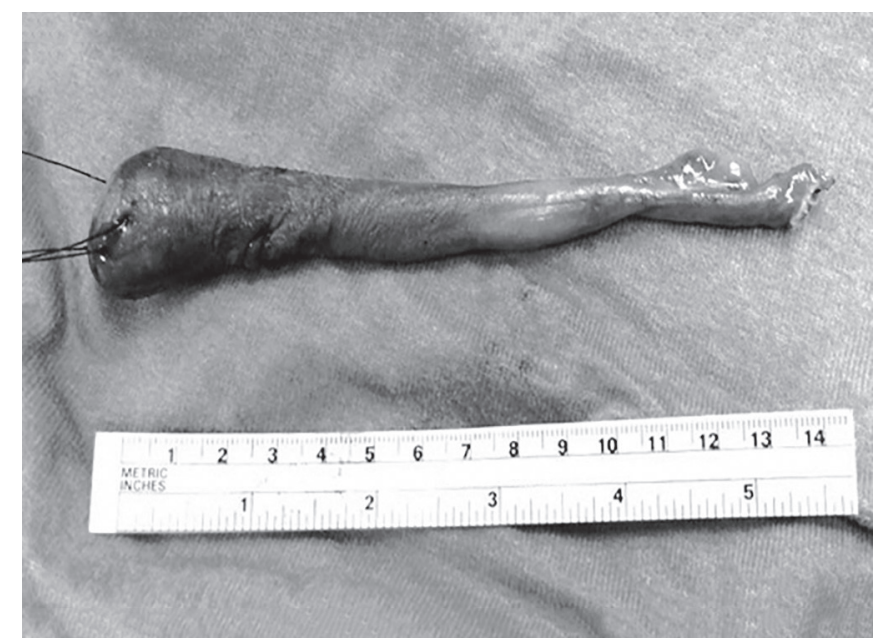

Fig. 3: Resected mass. The proximal part is on the right side and the distal part on the left 
dyspnea, dysphagia, persistent cough, or chest pain. The tumor is both diagnostically challenging and difficult to treat with gastroscopy in a single procedure. This report shows that a combined approach by a gastroenterologist and otolaryngologist, working cooperatively, can facilitate the correct diagnosis and provide optimum treatment.

\section{REFERENCES}

1. Shpitzer T, Noyek AM, Witterick I, Kassel T, Ichise M, Gullane P, Neligan P, Freeman J. Noncutaneous hemangiomas of the head and neck. Am J Otolaryngol 1997 Nov-Dec;18(6):367-374.

2. Moersch HJ, Harrington SW. Benign tumor of the esophagus. Ann Otol Rhinol Laryngol 1997;53:800-807.

3. Sogabe M, Taniki T, Fukui Y, Yoshida T, Okamoto K, Okita Y, Hayashi H, Kimuara E, Kimura Y, Onose Y, et al. A patient with esophageal hemangioma. J Med Invest 2006 Feb;53(1-2):177-182.
4. Tominaga K, Arakawa T, Ando K, Umeda S, Shiba M, Suzuki N, Watanabe T, Takaishi O, Fujiwara Y, Uchida T. Oesophageal cavernous haemangioma diagnosed histologically, not by endoscopic procedures. J Gastroenterol Hepatol $2000 \mathrm{Feb} ; 15(2): 215-219$.

5. Kim AW, Korst RJ, Port JL, Altorki NK, Lee PC. Giant cavernous hemangioma of the distal esophagus treated with esophagectomy. J Thorac Cardiovasc Surg 2007 Jun;133(6): 1665-1667.

6. Ramo OJ, Salo JA, Bardini R, Nemlander AT, Färkkilä M, Mattila SP. Treatment of submucosal hemangioma of the esophagus using a simultaneous video-assisted thoracoscopy and esophagoscopy: description of a new minimally invasive technique. Endoscopy 1997 Jun;29(5):S27-S28.

7. Feist JH, Siconolfi EP, Gilman E. Giant cavernous hemangioma of the esophagus. JAMA 1976 Mar 15;235(11):1146-1147.

8. Raza W, Nasir H, Ilahi F, Zafar Z. Esophageal haemangioma: a case report and review of literature. J Pak Med Assoc 2010 Apr;60(4):310-311. 